1 DOES YOUR PATIENT HAVE A TREATMENT ESCALATION PLAN? THINK, DISCUSS, DOCUMENT, VALIDATE

'Joanna Han Yen Ting*, ${ }^{1}$ Helen Greenfield, ${ }^{1}$ Eleanor Rogers, ${ }^{2}$ Ellie Topulli, ${ }^{2}$ Matteo Marino ${ }^{1}$ Gemma Savin, ${ }^{1}$ Saurabh Chaudhri. ${ }^{1}$ Department of Acute Medicine, Whipps Cross University Hospital, London, UK; ${ }^{2}$ Resuscitation Officer, Whipps Cross University Hospital, London, UK

\subsection{6/leader-2021-FMLM.1}

Introduction Evidence suggests that healthcare professionals are poor at initiating discussions with patients about treatment escalation plans (TEP), despite most patients wishing to be involved in this. Patients are often subject to futile resuscitation attempts or distressing investigations and treatment while doctors on-call struggle with hurried decisions in acute settings.

Aim To improve documentation of treatment escalation plans for medical patients in the Acute Assessment Unit (AAU) at a district general hospital in London.

Methods Leadership, communication and awareness of staff was identified as primary drivers for this project. Utilising Quality Improvement (QI) methodology, we ran Plan-DoStudy-Act (PDSA) cycles for each of our interventions.

We utilised the local Morbidity and Mortality meetings to highlight the importance of advanced care planning. During Covid simulation sessions in the pandemic, we placed strong emphasis on discussing TEP for acute admissions. Morning board meetings served as a 'checkpoint' to identify patients without a TEP. We designed a TEP poster which was displayed in AAU and circulated on social media. Emails were sent to consultants and junior doctors regarding the importance of discussing TEP.

We identified improvement by calculating the percentage of AAU patients with a documented TEP which was done by analysing electronic records weekly. We also recorded any inappropriate cardiac arrest calls based on data provided by the resuscitation team.

Results This project ran across 49 weeks and included 500 inpatients. The initial documentation rate of TEP was $40 \%$ at the start of the project and had risen to $70 \%$ by the end of the project.

Conclusions Healthcare professionals should be well supported in decisions surrounding appropriate TEP as this is highly complex. Effective leadership in this regard is crucial to ensure delivery of high-quality patient care.

*Presenter

\section{2 'THE TALE OF TWO COUNTRIES' MY PERSONAL OBSERVATION STORIES ON HOW TWO COUNTRIES HANDLED THE WAKE OF PANDEMIC FOCUSING ON ETHNIC MINORITY PEOPLE}

Lachhemi Rana. Unicaf University, UK, Hong Kong

10.1136/leader-2021-FMLM.2

Being present in two countries, Hong Kong and UK successively, when pandemic was just emerging, I was able to observe the wake of the pandemic and how it was handled especially by ethnic minority people in these two places. Hong Kong is a place, where wearing a mask in public area when a person is coughing or is sick is a normal sight to see. In terms of ethnic minority people, government tried to reach out to them by distributing pamphlets and making public awareness videos in their own languages which were distributed through community NGO's, volunteers and leaders who all worked together and did their best from their own side by distributing masks, sanitizers together with organizing health awareness programs. This maybe largely because a. everyone in Hong Kong who were present during SARS virus time already knew the drill and knew what they had to do despite the uncertainty b. public are well disciplined in terms of health c. community leaders connect with people and act like channel for government awareness program. Remarkably the most pronounced thing that I observed in all this was, just before the pandemic period, Hong Kong was going through political upheavals but much to everyone's surprise, Hong Kong people managed to pick themselves together keeping their political differences aside and came together to fight the pandemic first. Wearing mask and social distancing was still an unfamiliar site when I had just arrived in London. Local NGO workers, volunteers in London were seen distributing masks, sanitizers while restaurant people were also seen distributing free foods to ethnic minority people. Not everyone understood or followed the social distancing rules because this was totally new concept to everyone including those around them. I felt that border control took more time in UK and people were indecisive about the government's health plan. Overall, experience and discipline does help in getting better result when it comes to public health.

\section{$3 \quad$ 'SAMPARK' (COMMUNICATION) TO CONNECT WITH PATIENT'S FAMILY IN COVID TIMES}

Archana Bajaj, Shivani Arora. Max Super Speciality Hospital Shalimar Bagh, New Delhi, India

\subsection{6/leader-2021-FMLM.3}

The Novel Coronavirus posed a unique type of challenge that the healthcare industry across the globe faced. Despite the prevalent infection control practices and infrastructural controls pre-existing in the healthcare organizations, the virus demanded further changes not only in the infrastructure, but also in our day to day practices and behavioral patterns. In the journey of coming forth and delivering best care, satisfaction of patient attendants was a challenge. Due to COVID appropriate regulations, family was not able to meet their Loved ones during admission in hospital.

Problem Identification The Hospital Management along with senior clinicians looked into the root cause through Ishikawa Analysis .How the Patient satisfaction score decreased to $92 \%$ in IPD. Ishikawa Analysis churned up the reason for dissatisfaction . During difficult COVID times, families were already under lot of psychological stress and not being able to meet their loved ones who were admitted in the hospital further adding to their dissatisfaction.

Intervention Step 1 -In View of addressing the communication Barrier, a dedicated family liaison team was established. Step 2-At the time of patient admission, one dedicated SPOC and contact details of designate attendant was taken from the patient family to share a real time feedback with them. Step 3- Any challenge what so ever was escalated to Medical Admistration Team Step 4- ' Family Liaising Team' was in 